\title{
CHARGED PION PHOTOPRODUCTION FROM HYDROGEN AND DEUTERIUM AT JEFFERSON LAB
}

\author{
HAIYAN GAO* \\ on behalf of the Jefferson Lab \\ Hall A Collaboration, E94-104 Collaboration and E02-010 Collaboration
}

Laboratory for Nuclear Science and Department of Physics, Massachusetts Institute of Technology 77 Massachusetts Ave, Cambridge, MA 02139, U.S.A.

\begin{abstract}
The $\gamma n \rightarrow \pi^{-} p$ and $\gamma p \rightarrow \pi^{+} n$ reactions are essential probes of the transition from meson-nucleon degrees of freedom to quark-gluon degrees of freedom in exclusive processes. The cross sections of these processes are also advantageous, for the investigation of oscillatory behavior around the quark counting prediction, since they decrease relatively slower with energy compared with other photon-induced processes. Moreover, these photoreactions in nuclei can probe the QCD nuclear filtering and color transparency effects. In this talk, I discuss the preliminary results on the $\gamma p \rightarrow \pi^{+} n$ and $\gamma n \rightarrow \pi^{-} p$ processes at a center-of-mass angle of $90^{\circ}$ from Jefferson Lab experiment E94-104. I also discuss a new experiment in which singles $\gamma p \rightarrow \pi^{+} n$ measurement from hydrogen, and coincidence $\gamma n \rightarrow \pi^{-} p$ measurements at the quasifree kinematics from deuterium and ${ }^{12} \mathrm{C}$ for photon energies between $2.25 \mathrm{GeV}$ to $5.8 \mathrm{GeV}$ in fine steps at a center-of-mass angle of $90^{\circ}$ are planned. The proposed measurement will allow a detailed investigation of the oscillatory scaling behavior in photopion production processes and the study of the nuclear dependence of rather mysterious oscillations with energy that previous experiments have indicated. The various nuclear and perturbative QCD approaches, ranging from Glauber theory, to quark-counting, to Sudakov-corrected independent scattering, make dramatically different predictions for the experimental outcomes.
\end{abstract}

\section{Introduction}

Exclusive processes are essential to studies of transitions from the non-perturbative to the perturbative regime of Quantum Chromodynamics (QCD). The differential cross-section for many exclusive reactions ${ }^{1}$ at high energy and large momentum transfer appear to obey the quark counting rule. ${ }^{2}$ The quark counting rule was originally obtained based on dimensional analysis of typical renormalizable theories. The same rule was later obtained in a short-distance perturbative QCD approach by Brodsky and Lepage. ${ }^{3}$ Despite many successes, a model-independent test of the approach, called the hadron helicity conservation rule, tends not to agree with data in the similar energy and momentum region. The presence of helicity-violating amplitudes indicates that the short-distance expansion cannot be the whole story.

*Present address: Box 90305, Duke University, Durham, NC 27708, U.S.A. 
In addition some of the cross-section data can also be explained in terms of nonperturbative calculations. ${ }^{4}$

In recent years, a renewed trend has been observed in deuteron photo-disintegration experiments at SLAC and JLab. ${ }^{5,7}$ Onset of the scaling behavior has been observed in deuteron photo-disintegration ${ }^{7}$ at a surprisingly low momentum transfer of $1.0(\mathrm{GeV} / \mathrm{c})^{2}$ to the nucleon involved. However, a recent polarization measurement on deuteron photo-disintegration, ${ }^{8}$ carried out in Hall A at JLab, shows disagreement with hadron helicity conservation in the same kinematic region where the quark counting behavior is apparently observed. These paradoxes make it essential to understand the exact mechanism governing the early onset of scaling behavior.

Moreover, it is important to look closely at claims of agreement between the differential cross section data and the quark counting prediction. The elastic protonproton $(p p)$ scattering data at high energy and large momentum transfer has shown very interesting characteristics: the re-scaled $90^{\circ}$ center-of-mass angle data, $s^{10} \frac{d \sigma}{d t}$ show substantial oscillations about the power law behavior. With new high luminosity experimental facilities such as CEBAF, such oscillatory scaling behavior can be investigated with significantly improved precision in other exclusive processes. This will help identify the exact nature and the underlying mechanism governing the onset of the scaling behavior.

Oscillations are not restricted to the $p p$ sector; they are also seen in $\pi p$ fixed angle scattering. ${ }^{9}$ The situation for meson photoproduction is unsettled and needs urgent investigation. Rough power-law dependence of meson photoproduction seems to agree with the constituent quark counting rule prediction ${ }^{10}$ within experimental uncertainties, for example in the case of the $\gamma p \rightarrow \pi^{+} n$ process at a center-of-mass angle of $90^{\circ}$. Yet it is not clear whether the counting rule scaling behavior has been observed in the $\gamma p \rightarrow \pi^{0} p$ process because discrepancies exist between different measurements. For the $\gamma n \rightarrow \pi^{-} p$ process, no cross section data exist above a photon energy of $2.0 \mathrm{GeV}$ prior to the recent Jefferson Lab experiment E94-104, ${ }^{11}$ in which cross section measurements of this process from a deuterium target up to a photon energy of $5.6 \mathrm{GeV}$ have been carried out. Preliminary results indicate the constituent counting rule behavior in this channel at a center-of-mass angle of $90^{\circ}$, for photon energies above $\sim 3 \mathrm{GeV}$. In addition to the $\frac{1}{s^{7}}$ scaling behavior, these preliminary results suggest an oscillatory scaling behavior. The possible oscillatory scaling behavior needs to be investigated carefully, because the energy settings of E94-104 were designed to investigate the global constituent quark counting rule behavior, and thus chosen to be rather coarse.

The energy and nuclear dependence of such oscillatory behavior is also crucial in the search for signatures of the nuclear filtering and color transparency effects. The nuclear transparency of the $\gamma n \rightarrow \pi^{-} p$ process can be studied by taking the ratio of the pion photoproduction yield from a nuclear target such as ${ }^{12} \mathrm{C}$ to the yield from ${ }^{2} \mathrm{H}$. By finely mapping out the nuclear transparency over the scaling region it should be possible to test the nuclear filtering effect in a new regime.

We also note that the traditionally accepted "Glauber approximation" might be tested in the reactions under study. If the oscillations are a persistent feature of hard nucleon scattering, then established methods for obtaining the expected attenuation in nuclear targets exist. The qualitative nature of this approach is dramatically 
different from nuclear filtering. One way or the other, the experimental outcome of the photopion production from a nuclear target is expected to be of interest to a wide audience.

\section{Constituent Counting Rule and Oscillations}

The constituent counting rule predicts the energy dependence of the differential cross section at fixed center-of-mass angle for an exclusive two-body reaction at high energy and large momentum transfer as follows:

$$
d \sigma / d t=h\left(\theta_{c m}\right) / s^{n-2},
$$

where $s$ and $t$ are the Mandelstam variables, $s$ is the square of the total energy in the center-of-mass frame and $t$ is the momentum transfer squared in the $s$ channel. The quantity $n$ is the total number of elementary fields in the initial and final states, while $h\left(\theta_{c m}\right)$ depends on details of the dynamics of the process. In the case of pion photoproduction from a nucleon target, the quark counting rule predicts a $\frac{1}{s^{7}}$ scaling behavior for $\frac{d \sigma}{d t}$ at a fixed center-of-mass angle. The quark counting rule was originally obtained based on dimensional analysis under the assumptions that the only scales in the system are momenta and that composite hadrons can be replaced by point-like constituents. Implicit in these assumptions is the approximation that the class of diagrams, which represent on-shell independent scattering of pairs of constituent quarks (Landshoff diagrams) ${ }^{12}$ can be neglected. This counting rule was also confirmed within the framework of perturbative QCD analysis up to a logarithmic factor of $\alpha_{s}$ and are believed to be valid at high energy, in the perturbative $\mathrm{QCD}$ region. Such analysis relies on the factorization of the exclusive process into a hard scattering amplitude and a soft quark amplitude inside the hadron.

Although the quark counting rule agrees with data from a variety of exclusive processes, the other natural consequence of pQCD the hadron helicity conservation selection rule, tends not to agree with data in the experimentally tested region. Hadron helicity conservation arises from quark helicity conservation at high energies and the vector gluon-quark coupling nature of QCD, by neglecting the higher angular momentum states of quarks or gluons in hadrons. The same dimensional analysis which predicts the quark counting rule also predicts hadron helicity conservation for exclusive processes at high energy and large momentum transfers. If hadron helicity conservation holds, the induced polarization of the recoil proton in the unpolarized deuteron photo-disintegration process is expected to be zero. A polarization measurement ${ }^{8}$ in deuteron photo-disintegration has been carried out recently by the JLab E89-019 collaboration. While the induced polarization does seem to approach zero around a photon energy of $1.0 \mathrm{GeV}$ at $90^{\circ}$ center-of-mass angle, the polarization transfer data are inconsistent with hadron helicity conservation.

The entire subject is very controversial. Isgur and Llewellyn-Smith ${ }^{4}$ argue that if the nucleon wave-function has significant strength at low transverse quark momenta $\left(k_{\perp}\right)$, then the hard gluon exchange (essential to the perturbative approach) which redistributes the transfered momentum among the quarks, is no longer required.

\footnotetext{
†This point is currently under debate.
} 
The applicability of perturbative techniques at these low momentum transfers is in serious question. There are no definitive answers to the question- what is the energy threshold at which $p Q C D$ can be applied? Indeed the exact mechanism governing the observed quark counting rule behavior remains a mystery. Thus, it is crucial to look for other pQCD signatures as well.

Apart from the early onset of scaling and the disagreement with hadron helicity conservation rule, several other striking phenomena have been observed in $p p$ elastic scattering. One such phenomenon is the oscillation of the differential crosssection about the scaling behavior predicted by the quark counting rule $\left(s^{-10}\right.$ for $p p$ scattering), first pointed out by Hendry ${ }^{13}$ in 1973 . Secondly, the spin correlation experiment in $p p$ scattering first carried out at Argonne by Crabb et al. ${ }^{14}$ shows striking behavior: it is $\sim 4$ times more likely for protons to scatter when their spins are both parallel and normal to the scattering plane than when they are antiparallel, at the largest momentum transfers $\left(p_{T}^{2}=5.09(\mathrm{GeV} / \mathrm{c})^{2}, \theta_{c . m .}=90^{\circ}\right)$. Later spin-correlation experiments ${ }^{15}$ confirm the early observation by Crabb et al.. ${ }^{14}$ Theoretical interpretation for such an oscillatory behavior $\left(s^{10} \frac{d \sigma}{d t}\right)$ and the striking spin-correlation in $p p$ scattering was attempted by Brodsky, Carlson, and Lipkin ${ }^{16}$ within the framework of quantum chromodynamic quark and gluon interactions, where interference between hard pQCD short-distance and long-distance (Landshoff) amplitudes was discussed for the first time. The Landshoff amplitude arises due to multiple independent scattering between quark pairs in different hadrons. Although each scattering process is itself a short distance process, different independent scatterings can be far apart, limited only by the hadron size. Moreover, gluonic radiative corrections give rise to a phase to this amplitude which is calculable in pQCD. ${ }^{17}$ This effect is believed to be analogous to the Coulomb-nuclear interference that is observed in low-energy charged-particle scattering. It was also shown that at medium energies this phase (and thus the oscillation) is energy dependent, ${ }^{18}$ while becoming energy independent at asymptotically high energies. ${ }^{18,19}$

Lastly, Carroll et al. ${ }^{20}$ reported the anomalous energy dependence of nuclear transparency from the quasi-elastic $\mathrm{A}(\mathrm{p}, 2 \mathrm{p})$ process: the nuclear transparency first rises followed by a decrease. This intriguing result was confirmed recently at Brookhaven $^{21}$ with improved experimental technique in which the final-state was completely reconstructed. Ralston and Pire ${ }^{22}$ explained the free $p p$ oscillatory behavior in the scaled differential cross section and the $\mathrm{A}(\mathrm{p}, 2 \mathrm{p})$ nuclear transparency results using the ideas of interference between the short-distance and long-distance amplitudes and the QCD nuclear filtering effect. Carlson, Chachkhunashvili, and Myhrer $^{23}$ have also applied such an interference concept to the $p p$ scattering and have explained the $p p$ polarization data.

It was previously thought that the oscillatory $s^{10} \frac{d \sigma}{d t}$ feature is unique to $p p$ scattering or to hadron induced exclusive processes. However, it has been suggested that similar oscillations should occur in deuteron photo-disintegration, ${ }^{24}$ and photo-pion productions at large angles. ${ }^{25}$ The QCD re-scattering calculation of the deuteron photo-disintegration process by Frankfurt, Miller, Sargsian and Strikman ${ }^{24}$ predicts that the energy dependence of the differential cross-section, $s^{11} \frac{d \sigma}{d t}$ arises primarily from the $n-p$ scattering in the final state. If these predictions are correct, such oscillatory behavior may be a general feature of high energy exclusive photoreac- 
tions. Thus it is very important to experimentally search for these oscillations in photoreactions.

Farrar, Sterman and Zhang ${ }^{26}$ have shown that the Landshoff contributions are suppressed at leading-order in large-angle photoproduction but they can contribute at subleading order in $\frac{1}{Q}$ as pointed out by the same authors. In principle, the fluctuation of a photon into a $q \bar{q}$ in the initial state can contribute to independent scattering amplitude at sub-leading order. However, the vector-meson dominance diffractive mechanism is suppressed in vector meson photoproduction at large values of $t .^{27}$ On the other hand such independent scattering amplitude can contribute in the final state if more than one hadron exist in the final state, which is the case for both the deuteron photo-disintegration and nucleon photo-pion production reactions. Thus, an unambiguous observation of such an oscillatory behavior in exclusive photoreactions with hadrons in the final state at large $t$ may provide a signature of QCD final state interaction. The most recent data on $d(\gamma, p) n$ reaction ${ }^{7}$ show that the oscillations, if present, are very weak in this process. The rapid decrease of the cross section with energy $\left(\frac{d \sigma}{d t} \propto \frac{1}{s^{11}}\right)$ makes it impractical to investigate such oscillatory behavior.

Given that the nucleon photo-pion production has a much larger cross-section at high energies $\left(\frac{d \sigma}{d t} \propto \frac{1}{s^{7}}\right)$, it is very desirable to use these reactions to verify the existence of such oscillations. The preliminary $\gamma n \rightarrow \pi^{-} p$ and $\gamma p \rightarrow \pi^{+} n$ data from Experiment E94-104 with high statistical accuracy show hints of oscillation in the scaled differential cross-section. However, the rather coarse beam energy settings prevent a conclusive statement about the oscillatory behavior. Thus, to verify any structure in the scaled cross-section of photo-pion production processes, it is imperative that one performs a fine scan of the scaling region for the $\gamma p \rightarrow \pi^{+} n$ and the $\gamma n \rightarrow \pi^{-} p$ processes at a $90^{\circ}$ center-of-mass angle.

\section{Nuclear Filtering}

Nuclear filtering refers to the suppression of the long distance amplitude (Landshoff amplitude) in the strongly interacting nuclear environment. Large quark separations tend not to propagate in the nuclear medium while small quark separations propagate with small attenuation. This leads to suppression of the oscillation phenomena arising from the interference of the long distance amplitude with the short distance amplitude. Nuclear transparency measurements from $\mathrm{A}(\mathrm{p}, 2 \mathrm{p})$ experiments carried out at Brookhaven ${ }^{20}$ have shown a rise in transparency for $\mathrm{Q}^{2} \approx 3-8(\mathrm{GeV} / \mathrm{c})^{2}$, and a decrease in the transparency at higher momentum transfers. A more recent experiment, ${ }^{21}$ completely reconstructing the final-state of the $\mathrm{A}(\mathrm{p}, 2 \mathrm{p})$ reaction, confirms the validity of the earlier Brookhaven experiment. If the oscillatory behavior of the cross-section is suppressed in nuclei one would expect to see oscillations in the transparency, which are $180^{\circ}$ out of phase with the oscillations in the free $p p$ cross-section. On the other hand, Brodsky and de Teramond ${ }^{28}$ believed that the structure seen in $s^{10} \frac{d \sigma}{d t}(p p \rightarrow p p)$, the $A_{N N}$ spin correlation at $\sqrt{s} \sim 5 \mathrm{GeV}$ (around center-of-mass angle of $\left.90^{\circ}\right),{ }^{14,15}$ and the $A(p, 2 p)$ transparency result can be attributed to $c \bar{c} u u d u u d$ new resonant states. The opening of this channel gives rise to an amplitude with a phase shift similar to that predicted for gluonic radiative corrections. 
While interpretations of the elastic $p p \rightarrow p p$ cross section, the analyzing power $A_{N N}$ and the transparency data remain controversial, the ideas of nuclear filtering effect and the interference between the hard pQCD short-distance and the longdistance Landshoff amplitudes by Ralston and Pire ${ }^{22}$ are able to explain both the $s^{10} \frac{d \sigma}{d t}(p p \rightarrow p p)$ oscillatory behavior and the Brookhaven $\mathrm{A}(\mathrm{p}, 2 \mathrm{p})$ transparency data. Carlson, Chachkhunashvili, and $\mathrm{Myhrer}^{23}$ have also applied such an interference concept to explain the $p p$ polarization data.

Recently, a first complete calculation of "color transparency" and "nuclear filtering' in perturbative QCD has been carried out for electro-production experiments. ${ }^{29}$ These calculations show that the nuclear filtering effect is complementary to color transparency (CT) effect. Color transparency, first conjectured by Mueller and Brodsky $^{30}$ refers to the suppression of final (and initial) state interactions of hadrons with the nuclear medium in exclusive processes at large momentum transfers. The phenomenon of CT occurs when exclusive processes proceed via the selection of hadrons in the so-called point-like-configuration (PLC) states. Furthermore this small configuration should be "color screened" outside its small radius and the compact size should be maintained while it traverses the nuclear medium. While nuclear filtering uses the nuclear medium actively, in CT large momentum transfers select out the short distance amplitude which are then free to propagate through the passive nuclear medium. The expansion time relative to the time to traverse the nucleus is an essential factor for the observation of the CT effect, based on the quantum diffusion model by Farrar, Liu, Frankfurt and Strikman. ${ }^{31}$ Thus, while one expects to observe the onset of CT effect sooner in light nuclei compared to heavier nuclei, the large A limit provides a perturbatively calculable limit for the nuclear filtering effect. This makes ${ }^{12} \mathrm{C}$ a good choice as the target for the proposed nuclear transparency measurement. The experimental verification of the nuclear filtering effect would be a very interesting confirmation of this QCD based approach in the transition region. For a detailed discussion on the nuclear filtering effect and related subjects, we refer to a review article on the subject. ${ }^{32}$

\section{Experiment E94-104 and Future Extension}

Experiment E94-104 was carried out in Hall $\mathrm{A}^{34}$ at the Thomas Jefferson National Accelerator Facility (JLab). The continuous electron beam, at a current around $30 \mu \mathrm{A}$ and energies from 1.1 to $5.5 \mathrm{GeV}$, impinged on a $6 \%$ copper radiator and generated an untagged bremsstrahlung photon beam. The production data were taken with the $15 \mathrm{~cm}$ cryogenic liquid hydrogen (LH2) target for singles $p\left(\gamma, \pi^{+}\right) n$ measurement, or with the liquid deuterium (LD2) target for coincidence $d\left(\gamma, \pi^{-} p\right) p$ measurement. The background was measured by recording data without the production target and with or without the radiator. The two High Resolution Spectrometers (HRS) in Hall A, with a momentum resolution of better than $2 \times 10^{-4}$ and a horizontal angular resolution of better than $2 \mathrm{mrad}$, were used to detect the outgoing pions and recoil protons. Based on two-body kinematics, the incident photon energy was reconstructed from final states, i.e. the momentum and angle of the $\pi^{+}$in the singles measurement, momenta and angles of the $\pi^{-}$and $p$ in the coincidence measurement. Both spectrometers consisted of magnets to focus and bend the charged particles $\left(45^{\circ}\right)$, vertical drift chambers (VDCs) to record the tracks, 
and scintillator planes (S1/S2) to generate triggers. Two new aerogel Čerenkov detectors (A1/A2) in the left spectrometer provided particle identification for positive particles, mainly pions and protons, since the time-of-flight technique fails at high momentum. The $\mathrm{CO}_{2}$ gas Cerenkov detector and preshower/shower detector provided particle identification for negative particles, mainly pions and electrons.

The photon energies were reconstructed from final states by assuming exclusive single-pion production. A $100 \mathrm{MeV}$ bin with the middle $75 \mathrm{MeV}$ from the beam energy, was chosen for the data analysis, where the multi-pion contribution was negligible. The data after background subtraction, with cuts on trigger type, coincidence timing, PID (particle identification), acceptance and photon energy, were compared to the Monte Carlo simulation with the same cuts on acceptance and photon energy. The raw cross section was extracted by comparing data and simulation. The simulation was performed with a modified JLab Hall A Monte Carlo program, MCEEP. ${ }^{35}$ The bremsstrahlung photon flux was calculated with an estimated $3 \%$ uncertainty, by using the thick-radiator codes written by Meekins, ${ }^{36}$ based on the formulae of Matthews et al. ${ }^{37}$ The momentum distribution of neutrons in deuterium target as well as its binding energy was considered in the simulation. Different calculations of the momentum distribution were tried and little model dependence was found $(<1 \%)$. The angular distribution input for the cross section was obtained by fitting the $\pi^{+}$photoproduction data at 4,5 and $7.5 \mathrm{GeV} .^{10}$ It was used for all the kinematics including the $\pi^{-}$photoproduction since the extraction of the cross section at $\theta_{c m}=90^{\circ}$ was insensitive to the angular distribution $(<1 \%)$. The distributions of acceptance, reconstructed momentum and photon energy were in good agreement with results obtained from simulations.

Important correction factors were applied to deduce the final cross section. The nuclear transparency in the deuteron due to the final state interaction led to a correction of approximately $20 \%$. The correction due to material absorption was applied to compensate for the scattering losses in the target and the spectrometer. The correction for a single pion or proton was approximately $6 \%$, with major losses in the target, scintillators and aerogel. The computer dead time correction was considered on a run-by-run basis and was at most a few percent. The pion decay loss was calculated from the pion flight path length. An additional correction, approximately $2 \%$, was applied since some muons from pion decay may be detected and mis-indentified as pions. The detector inefficencies also led to some corrections, mostly less than $1 \%$. The total errors were dominated by systematic uncertainties and were estimated to be $8 \%$ in cross section, while point-to-point uncertainties for the three kinematics at $3.3,4.2$ and $5.5 \mathrm{GeV}$ to be $4 \%$. The statistical errors were approximately $2 \%$.

Fig. 1 shows the results of the scaled differential cross section $\left(s^{7} \frac{d \sigma}{d t}\right)$ for the $\gamma p \rightarrow \pi^{+} n$ process at $\theta_{c m}=90^{\circ}$. The new results with fitted value $n=8.9 \pm 0.2$ (see Eq. 1) agree with those of Anderson et al. ${ }^{10}$ and exhibit the scaling behavior predicted by the constituent counting rule with 9 elementary fields. The lowest energy datum in the inset box of Fig. 1 corresponds to a center-of-mass energy of approximately $2.7 \mathrm{GeV}$ and photon energy of $3.3 \mathrm{GeV}$.

Fig. 2 shows the results of the scaled differential cross section $\left(s^{7} \frac{d \sigma}{d t}\right)$ for the $\gamma n \rightarrow \pi^{-} p$ process at $\theta_{c m}=90^{\circ}$. The new results greatly extend the existing 


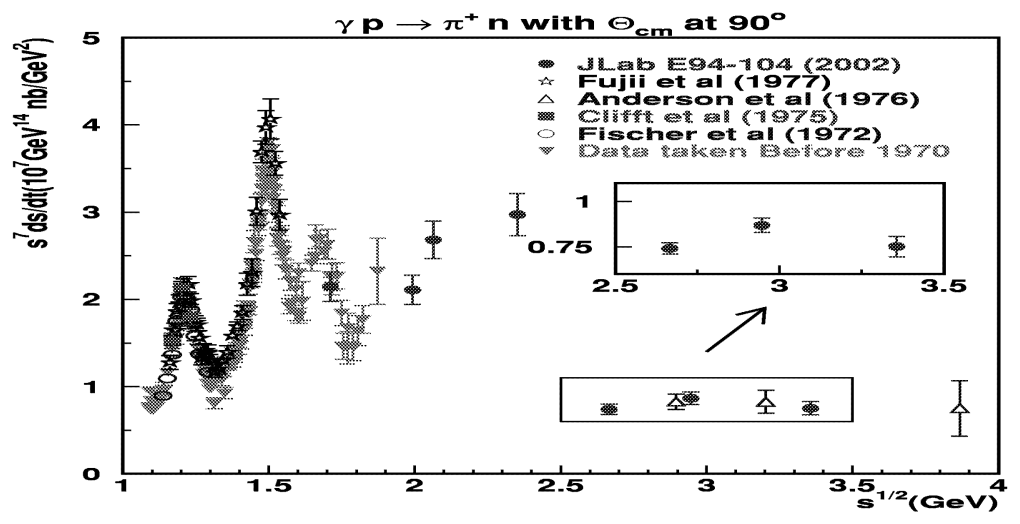

Fig. 1. The scaled differential cross section $s^{7} \frac{d \sigma}{d t}$ versus center-of-mass energy for the $\gamma p \rightarrow \pi^{+} n$ at $\theta_{c m}=90^{\circ}$. The data from JLab E94-104 are shown as solid circles. The error bars for the new data and Anderson et al.'s data, ${ }^{10}$ include statistical and systematic uncertainties, except those in the inset in which only point-to-point systematic uncertainties are included to highlight the possible oscillatory scaling behavior. Other data sets ${ }^{38,39}$ are shown with statistical errors only.

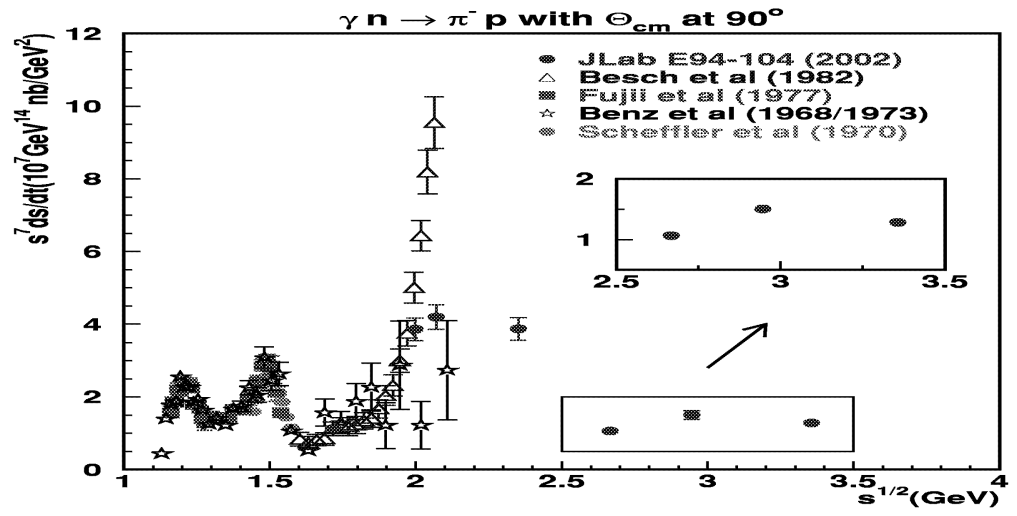

Fig. 2. The scaled differential cross section $s^{7} \frac{d \sigma}{d t}$ versus center-of-mass energy for the $\gamma n \rightarrow \pi^{-} p$ at $\theta_{c m}=90^{\circ}$. The data from JLab E94-104 are shown as solid circles. The error bars for the new data include statistical and systematic uncertainties, except those in the inset in which only point-to-point systematic uncertainties are included to highlight the possible oscillatory scaling behavior. 
measurements and exhibit, for the first time, a global scaling behavior at high energy for this reaction with fitted value $n=8.6 \pm 0.2$. The scaling behavior in $\pi^{-}$ production is similar to that in $\pi^{+}$production, except for possibly more pronounced oscillations in $\pi^{-}$channel, as suggested by the insets in Fig. 2. Unfortunately, the coarse energy setting of this experiment does not allow a conclusive statement on the oscillatory scaling behavior.

Recently a new experiment ${ }^{41}$ was proposed to carry out a measurement of the photo-pion production cross-section for the fundamental $\gamma n \rightarrow \pi^{-} p$ process from a ${ }^{2} \mathrm{H}$ and ${ }^{12} \mathrm{C}$ target and for the $\gamma p \rightarrow \pi^{+} n$ process from a hydrogen target at a centerof-mass angle of $90^{\circ}$, at $\sqrt{s} \sim 2.25 \mathrm{GeV}$ to $3.41 \mathrm{GeV}$ in steps of approximately 0.07 $\mathrm{GeV}$. The nuclear transparency for the $\gamma n \rightarrow \pi^{-} p$ process will be formed by taking the cross-section ratio of ${ }^{12} \mathrm{C}$ to that of ${ }^{2} \mathrm{H}$. The new experiment will make individual cross-section measurement with a $2 \%$ statistical uncertainty and a point-to-point systematic uncertainty of $<3 \%$. Such precision will allow the test of the oscillatory behavior in the scaled fundamental cross section measurement. The systematic uncertainties for the transparency measurement will be greatly reduced when one takes the ratio of carbon to ${ }^{2} \mathrm{H}$. Thus, the proposed transparency measurement is expected to have combined statistical and systematic uncertainties of $<5 \%$, which should be sufficient to provide evidence for or against the nuclear filtering effect in nuclear photo-pion production processes. With combined statistical and systematic uncertainties of $<5 \%$, it should be possible to test the Glauber predictions as well. The proposed experiment will only be possible with the unique JLab capability of high luminosity and such an experiment will be carried out in Hall A at JLab.

In summary, the preliminary data from E94-104 with $E_{\gamma} \geq 3.3 \mathrm{GeV}$ exhibit a global scaling behavior in both $\pi^{-}$and $\pi^{+}$photoproduction, consistent with the constituent counting rule. Furthermore, E94-104 results in a rather coarse step of $\sqrt{s}$, seem to suggest oscillatory behavior in $s^{7} \frac{d \sigma}{d t}$. Thus, it is essential to confirm such oscillatory behavior in finer step of $\sqrt{s}$ in the $\gamma p \rightarrow \pi^{+} n$ and the $\gamma n \rightarrow \pi^{-} p$ processes. Furthermore, a nuclear transparency measurement of the $\gamma n \rightarrow \pi^{-} p$ process from a ${ }^{12} \mathrm{C}$ target will allow the search of the nuclear filtering effect. Such an experiment is currently being planned at JLab.

\section{Acknowledgments}

This work is supported in part by the U.S. Department of Energy under contract number DE-FC02-94ER40818.

\section{References}

1. G. White et al., Phys. Rev. D49, 58 (1994).

2. S.J. Brodsky and G.R. Farrar, Phys. Rev. Lett. 31, 1153 (1973); Phys. Rev. D11, 1309 (1975); V. Matveev et al., Nuovo Cimento Lett. 7, 719 (1973);

3. G.P. Lepage, and S.J. Brodsky, Phys. Rev. D22, 2157 (1980).

4. N. Isgur and C. Llewelyn-Smith, Phys. Rev. Lett. 52, 1080 (1984).

5. J. Napolitano et al., Phys. Rev. Lett. 61, 2530 (1988); S.J. Freedman et al., Phys. Rev. C48, 1864 (1993); J.E. Belz et al., Phys. Rev. Lett. 74, 646 (1995).

6. C. Bochna et al., Phys. Rev. Lett. 81, 4576 (1998). 
7. E.C. Schulte, et al., Phys. Rev. Lett. 87, 102302 (2001);

8. K. Wijesooriya, et al., Phys. Rev. Lett. 86, 2975 (2001).

9. D. P. Owen et al., Phys. Rev. 181, 1794 (1969); K. A. Jenkins et al., Phys. Rev. D21, 2445 (1980); C. Haglin et al., Nucl. Phys. B216, 1 (1983).

10. R.L. Anderson et al., Phys. Rev. D14, 679 (1976).

11. Jefferson Lab Experiment E94-104, Spokespersons: H. Gao, R.J. Holt.

12. P. V. Landshoff, Phys. Rev. D10, 1024 (1974).

13. A.W. Hendry, Phys. Rev. D10, 2300 (1974).

14. D.G. Crabb et al., Phys. Rev. Lett. 41, 1257 (1978).

15. G.R. Court et al., Phys. Rev. Lett. 57, 507 (1986), T.S. Bhatia et al., Phys. Rev. Lett. 49, 1135 (1982), E.A. Crosbie et al., Phys. Rev. D23, 600 (1981).

16. S.J. Brodsky, C.E. Carlson, and H. Lipkin, Phys. Rev. D20, 2278 (1979).

17. A. Sen, Phys. Rev. D28, 860 (1983).

18. J. Botts and G. Sterman, Nucl. Phys. B325, 62 (1989).

19. A. H. Mueller, Phys. Rep. 73, 237 (1981).

20. A.S. Carroll et al., Phys. Rev. Lett. 61, 1698 (1988).

21. Y. Mardor et al., Phys. Rev. Lett. 81, 5085 (1998); A. Leksanov et al., Phys. Rev. Lett. 87, 212301-1 (2001).

22. J.P. Ralston and B. Pire, Phys. Rev. Lett. 61, 1823 (1988), J.P. Ralston and B. Pire, Phys. Rev. Lett. 65, 2343 (1990).

23. C.E. Carlson, M. Chachkhunashvili, and F. Myhrer, Phys. Rev. D46, 2891 (1992).

24. L.L. Frankfurt, G.A. Miller, M.M. Sargsian, and M.I. Strikman, Phys. Rev. Lett. 84, 3045 (2000); M.M. Sargsian, private communication.

25. P. Jain, B. Kundu, and J. Ralston, hep-ph/0005126.

26. G.R. Farrar, G. Sterman, and H. Zhang, Phys. Rev. Lett. 62, 2229 (1989).

27. E. Anciant et al., Phys. Rev. Lett. 85, 4682 (2000).

28. S. J. Brodsky, and G. F. de Teramond, Phys. Rev. Lett. 60, 1924 (1988).

29. B. Kundu, J. Samuelsson, P. Jain and J.P. Ralston, Phys. Rev. D62, 113009 (2000).

30. S.J. Brodsky and A.H. Mueller, Phys. Lett. B 206, 685 (1988).

31. G.R. Farrar, H. Liu, L.L. Frankfurt, and M.I. Strikman, Phys. Rev. Lett. 61, 686 (1988).

32. P. Jain, B. Pire, and J.P. Ralston, Phys. Rep. 271, 67 (1996).

33. P. Jain, private communications.

34. B. D. Anderson et al., to be submitted to Nucl. Instrum. Methods.

35. P. Ulmer et al., http://www.physics.odu.edu/ ulmer/mceep/mceep.html.

36. D. G. Meekins, Ph.D. Thesis, College of William and Mary, unpublished, (1998).

37. J. L. Matthews and R. O. Owens, Nucl. Instrum. Methods 111, 157 (1973); J. L. Matthews, D. J. S. Findlay and R. O. Owens, Nucl. Instrum. Methods 180, 573 (1981).

38. K.-H. Hellwege, Numerical Data and Functional Relationships in Science and Technology, Group I, Volume 8: H. Genzel, P. Joos and W. Pfeil, Photoproduction of Elementary Particles, (1973).

39. HEPDATA: REACTION DATA Database, http://durpdg.dur.ac.uk/hepdata/reac.html.

40. H.-J. Besch et al., Z. Phys. C16, 1 (1982).

41. Jefferson Lab Proposal PR02-010, Spokespersons: D. Dutta, H. Gao and R.J. Holt. 